\title{
Potentiation of Viral Carcinogenesis by Immunosuppression
}

\author{
A. C. ALLISON,* M.SC., D.PHIL., B.M.
}

The role of immunity in controlling the development of tumours may be conveniently analysed by comparing the incidence of tumours induced by viruses in normal animals with that in immunosuppressed animals. The immunosuppressive procedures used have included thymectomy, treatment with antilymphocytic serum, or both. Any of these methods inhibit cell-mediated immunity and have definite effects on the production of tumours by viruses.

The most fully studied example is polyoma virus, which is a small DNA-containing virus that infects many laboratory and wild mouse colonies. ${ }^{1}$ This virus has also been used in molecular biological studies of viral transformation of cells in vitro. ${ }^{2}$ Natural infections by polyoma virus are transmitted horizontally in mouse colonies. Virus is not normally transmitted from mothers to offspring; indeed, the latter are temporarily protected from infection by neutralizing antibody acquired from their mothers. Tumours are hardly ever seen in colony infections, though inoculation of relatively large doses of virus into newborn mice of susceptible strains produces a high incidence of tumours. With resistant strains inoculation of large doses of virus into newborns results in the formation of tumours in only relatively few animals. Tumours are hardly ever seen if polyoma virus-even in enormous doses-is inoculated into adult mice of any strain.

\section{Low Incidence of Adult Tumours}

Several questions can now be asked. Firstly, is the low incidence of tumours in adult mice infected with polyoma virus due to failure of transformation by the virus or to the fact that adult animals can mount an effective immune response against polyoma tumours? The latter explanation can now be taken as correct. $\mathrm{Law}^{3}$ and Taylor and $\mathrm{I}^{4}$ independently found polyoma tumours in thymectomized mature mice after colony infections. Non-thymectomized mice in the same colonies were infected with polyoma virus, as shown by the presence of antibodies, but did not develop tumours. Gaugas et al..$^{5}$ reported an "epidemic" of polyoma tumours in thymectomized mice inoculated with human leprosy bacilli and antilymphocytic serum after an infection of the animal house with polyoma virus.

Because antilymphocytic serum (A.L.S.) might potentiate the multiplication of virus in other ways than by suppressing cellmediated immunity (for example, by stimulating proliferation of lymphoblasts, in which viruses multiply more readily than in untransformed lymphocytes, or by interfering with macrophage function), and because leprosy organisms might themselves have an adjuvant or immunosuppressive effect, ${ }^{6}$ there was some doubt about the interpretation of these interesting observations. We have therefore performed better controlled experiments with CBA mice.

In these thymectomy was performed at 6 weeks of age; they were then infected with polyoma virus and given weekly injections of antilymphocytie serum. These injections were continued for seven weeks, when the first tumours appeared; no further antilvmphocytic serum was given. Ten days later the mice were divided into three groups. The first group was left untouched. In the second immunocompetence was restored with syngeneic (that is, genetically compatible) adult lymphoid cells. In the third group the restoration was made with lymphoid cells from syngeneic mice

- Head of the Division of Cell Pathology, Clinical Research Centre, Northwick Park, Harrow, Middlesex. already sensitized against the antigens of the polyoma tumour. No tumours developed in the last group; all but one of the second group and all of the first group developed polyoma tumours. The failure of the unsensitized lymphoid cells to protect is almost certainly due to the long interval after virus inoculation. Other experiments show that they can protect if given about one week after virus inoculation to sensitized animals.

These experiments provide strong evidence that adult mice are protected because they can mount an effective cellmediated immune response against polyoma tumours. If immunosuppression is efficient (by a combination of thymectomy and the administration of antilymphocytic serum) all the mice develop polyoma tumours. Small tumours were excised; if the animals were then given lymph node cells from syngeneic animals sensitized against the specific tumour antigen no tumours recurred, whereas without this treatment the tumours always recurred. These results show that immunotherapy may be a highly effective adjunct to surgery; but the difference from the situation in man needs no stressing.

\section{Inherited Differences}

The second question concerns the inherited differences in susceptibility of different mouse strains to tumour induction by polyoma virus. Are these due to the ease with which cells can be transformed by the virus or to differences in immune responses? Transformation in vitro of cells from the resistant C57BL strain by polyoma virus occurs as readily as transformation of cells from sensitive strains. Thymectomized or A.L.S.-treated C57BL mice infected in the neonatal period with polyoma virus all develop polyoma tumours, ${ }^{7}$ and I have found that thymectomized, A.L.S.-treated adult mice of this strain develop tumours after colony infection with the virus. Thus in this case the genetically controlled resistance is due to precocious development of cell-mediated immunity against the tumour in resistant strains, and can be overcome by immunosuppression.

Thirdly, is the susceptibility of newborn animals to virus oncogenesis a result of their becoming tolerant to the tumour antigen? This is not the case. ${ }^{8}$

Adult and newborn mice were inoculated with polyoma virus; ten days and six weeks later their lymphoid cells were tested for sensitization to the virus. This was done by transferring some cells to syngeneic thymectomized animals which had been inoculated with polvoma virus. The lymphoid cells from the adults prevented the development of tumours in the recipients. Cells from the newborns prevented this when taken six weeks after the inoculation of virus, but not when taken ten days after this. Thus in adults cellmediated immunity is rapidly mounted, being fully established by ten days after the administration of virus (probably earlier), whereas in the newborn cell-mediated immunity develops slowly and is therefore unable to check tumour development.

Even when viruses are transmitted from mother to offspring tolerance to tumour-specific antigens does not develop. We were therefore led to re-examine the classical experiments of Billingham and his colleagues ${ }^{910}$ on the induction of tolerance by inoculating foreign cells into newborns. Lymphocytes from the "tolerant" animals, in which skin grafts from the donor strain could be permanently accepted, had the capacity to react in vitro against target cells from the donor strain, while their sera specifically blocked this reaction." Hence, it seems very difficult to produce tolerance 
against cellular-including tumour-antigens, and probably many cases of supposed tolerance are due to blocking or enhancing antibodies.

\section{Leukaemogenic Viruses}

The second group of naturally occurring oncogenic viruses in mice is the leukaemogenic viruses. Most mouse strains carry leukaemogenic viruses related to Gross virus, which are transmitted vertically from mothers (and sometimes from fathers) to offspring. For many years it was thought that animals were tolerant to such viruses, producing neither antibody nor cell-mediated immunity. If that were the case, immunosuppression would not be expected to affect tumour development. In fact, it does. Thymectomy cannot be used in this situation, because the production of leukaemia by both the Gross and the Moloney virus requires the presence of a thymus. However, Allison and $\mathrm{Law}^{7}$ found that Moloney lymphoma or leukaemia was greatly potentiated by inoculations of antilymphocytic serum. We have found that the latent period for leukaemia induction in AKR mice, which carry the Gross virus, is shortened by the administration of antilymphocytic serum.

Wahren and Metcalfe ${ }^{12}$ have found that lymphocytes from AKR mice are cytotoxic to target cells carrying the Gross virus, so that they can no longer be regarded as tolerant. Other evidence that this is so has been presented by Dore et $a l .^{13}$ In this case nearly all animals left long enough develop leukaemia (after a latent period of about 300 days). Immunity does not prevent leukaemia but delays its onset, which allows the animals to breed before onset of malignancy. Immunosuppression accelerates the malignancy.

\section{Immunological Surveillance}

All these results leave little doubt that the immunological surveillance mechanism operates very efficiently against virusinduced tumours in animals, either preventing or delaying the onset of malignancy. All the tumours so far found in mice receiving long-term immunosuppression have been leukaemias, lymphomas, or mammary tumours, or polyomainduced tumours of the salivary glands or osteosarcomas. In all these cases there is reason to believe that a virus may be concerned in the aetiology. Though the surveillance mechanism may also operate against tumours not induced by viruses, there is no direct evidence that this is the case. Nevertheless, it would be premature to conclude that the tumours occurring in human patients treated with immunosuppressants are virus-induced, though the parallel between animals is striking enough to demand further analysis.

\section{REFERENCES}

1 Huebener, R. J., Harvey Lectures, 1961, 56, 45.

Dulbecco, R., Science, 1969, 166, 962.

${ }^{3}$ Law, L. W., Cancer Research, 1966, 26, 551.

4 Allison, A. C., and Taylor, R. B., Cancer Research, 1967, 27, 703.

5 Gaugas, J. M., et al., Nature, 1969, 221, 1033.

s Ptak, W., Gaugas, J. M., Rees, R. J. W., and Allison, A. C., Clinical and Experimental Immunology, 1970, 6, 117 .

Allison, A. C., and Law, L. W., Proceedings of the Society for Experimental Biology and Medicine, 1968, 127, 207.

s Allison, A. C., in Proceedings of the Fourth Quadrennial Cancer Conference, Perugia, p. 376, 1970.

9 Billingham, R. E., Brent, L., and Medawar, P. B., Nature, 1953, 172, 603. 10 Billingham, R. E., and Brent, L., Proceedings of the Royal Society (Series B), 1956, 146, 78 .

11 Hellström, I., Allison, A. C., and Hellström, K. E., Nature. In press.

12 Wahren, B., and Metcalfe, D., in Proceedings of the Fourth Quadrennial Cancer Conference, Perugia, p. 473, 1970.

13 Doré, J. F., Ajuria, E., and Mathé, G. Revue Européenne d'Études Cliniques et Biologiques, 1970, 15, 8.

\title{
Immunosurveillance and Cancer : Epidemiological Evidence
}

\author{
R. DOLL, ${ }^{*}$ o.B.E., F.R.C.P., F.R.S., and L. KINLEN, $†$ M.B., B.S., M.R.C.P.
}

The mechanism by which cancer develops remains one of the great mysteries of modern medicine. The concept that it results from a breakdown in "immunological surveillance," which permits the proliferation of an abnormal clone of cells with a selective advantage over other cells, has been widely welcomed as a stimulus to thought and experiment. Keast ${ }^{1}$ has recently summarized the evidence for this hypothesis under four heads. Firstly, cancer in man occurs characteristically at the extremes of life when the immune system is either maturing or is weakened by thymic atrophy. Secondly, its incidence is increased following the use of immunosuppressive drugs. Thirdly, the same effect is produced experimentally by thymectomy in mice. Fourthly, the rare diseases that involve a deficiency of cell-mediated immunity are characterized by a high incidence of tumours in those subjects who survive long enough to allow their development.

In the present paper we review only those parts of the evidence that have been obtained from observations on man.

\section{Immunological Disorders}

Consider first the frequency with which cancer has been reported in association with immunological disorders. These include three congenital disorders-ataxia-telangiectasia and the Wiskott-Aldrich and Chediak-Higashi syndromes.

\footnotetext{
* Regius Professor of Medicine.

t Lecturer in Medicine.

Department of the Regius Professor of Medicine, Radcliffe Infirmary, Oxford.
}

The first is an autosomal recessive trait characterized by progressive cerebellar ataxia in childhood, telangiectases (particularly in the conjunctiva), and an increased susceptibility to respiratory infections. Malignant tumours have been recorded in no fewer that 14 out of the 200 cases reported, including five reticulum cell sarcomas, two cases of Hodgkin's disease, two lymphosarcomas, and two gastric carcinomas. The immunological defect has not been specified, but it involves defective cellular immunity and antibody response to antigen and shows abnormalities in the level of serum immunoglobulin and in the thymus. ${ }^{2}$

The Wiskott-Aldrich syndrome affects boys, producing thrombocytopenia, eczema, and recurrent infections. Most die early from haemorrhage or infection, but despite this malignant tumours, mainly reticuloendothelioses, were present in 11 of the 90 reported cases. Immunoglobulin levels, cellular immunity, and antibody response to antigen are initially normal, but subsequently the serum immunoglobulin decreases and small lymphocytes disappear from the circulation and the lymph nodes. ${ }^{3}$

The Chediak-Higashi syndrome is an autosomal recessive trait, characterized by distinctive granule-containing leucocytes, a pigment defect in the skin, hair, and eyes, and again a pronounced susceptibility to infection. In at least 11 out of the 50 reported cases a terminal illness has developed in which the appearance of the lymphoid tissue suggested a lymphoma. The nature of the immunological deficiency has not yet been discovered. ${ }^{4}$

A fourth congenital disorder, agammaglobulinaemia, is better considered together with hypogammaglobulinaemia. These conditions, when not due to some other disease, present a wide variety of clinical and pathological features. Cases with onset in infancy tend to be in males with a positive family history, but this is not an absolute rule and the classification of individual cases may be extremely difficult. Many cases that appear to be acquired may 